Received: 2015.05 .07
Accepted: 2015.05 .31
Published: 2015.08 .01
Authors' Contribution:
A Study Design
B Data Collection
C Statistical Analysis
D Data Interpretation
E Manuscript Preparation
F Literature Search
G Funds Collection

\title{
Trifurcation of the Left Common Carotid Artery
}

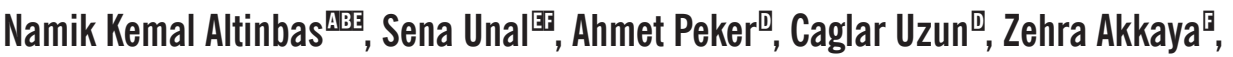 Elif Peker ${ }^{\text {B }}$}

\author{
Department of Radiology, Ankara University Faculty of Medicine, Ibni Sina Hospital, Ankara, Turkey
}

Author's address: Namik Kemal Altinbas, Department of Radiology, Ankara University Faculty of Medicine, Ibni Sina Hospital, Ankara, Turkey, e-mail: namikaltin@gmail.com

\section{Summary}

Background: Trifurcation of the common carotid artery is an unusual variation.

Case Report: $\quad$ We report a case of left common carotid artery trifurcation in a 74-year-old man. The left common carotid artery divided into the internal carotid, external carotid and facial arteries. Herein, the anatomy of the carotid arteries and the Doppler sonography and CT angiography findings of the left common carotid artery trifurcation were described with images.

Concluisons:

The variations of the carotid arteries should be known to avoid and reduce the complications during the invasive procedures.

\section{MeSH Keywords: $\quad$ Anatomic Variation • Carotid Artery Diseases • Carotid Artery, Common \\ PDF file: $\quad$ http://www.polradiol.com/abstract/index/idArt/894579}

\section{Background}

Common carotid artery trifurcation is a rare anatomic variation of the terminal portion of the common carotid artery. Normally common carotid arteries bifurcate into an internal carotid artery and an external carotid artery at the level of fourth cervical vertebra or the upper border of the thyroid cartilage at the both sides [1-3]. From time to time, however, variations of the common carotid artery can be seen. In some cases the common carotid artery can terminate as the external and internal carotid arteries and another artery, which is normally a branch of the external carotid artery or the subclavian artery. In some other cases high or low bifurcation of the common carotid artery can occur as another variation and even though it is very rare, the absence of common carotid artery also can be seen $[1,4]$. It is important to know the variations of the carotid arteries before applying invasive procedures to avoid complications [2,3]. We present a case with a trifurcation of the left common carotid artery, which terminates as the internal carotid artery, the external carotid artery and the facial artery.

\section{Case Report}

A 74-year-old man was admitted to the department of peripheral vascular surgery with a history of non-healing wounds of the left foot. The patient had hypertension for
10 years, diabetes mellitus for 13 years and chronic renal disease for 1.5 years. Doppler ultrasonography (US) of the carotid arteries and digital subtraction angiography of the lower extremity arteries were performed for peripheral vascular disease as a preliminary diagnosis. The carotid artery Doppler US revealed thickening of the intima-media at both sides, bilateral non-stenotic soft plaques of the carotid bifurcations and the trifurcation of the left common carotid artery. For detailed examination of the carotid arteries, computed tomography (CT) angiography was performed. The examination also showed non-stenotic soft plaques of the carotid arteries, trifurcation of the left common carotid artery and about $50-60 \%$ stenosis of the left vertebral artery. CT angiography demonstrated trifurcation of the left common carotid artery at the level of superior margin of the fourth cervical vertebra and the inferior margin of the hyoid bone. The left common carotid artery terminated as the internal carotid artery, the external carotid artery and the facial artery (Figures 1-3). The right common carotid artery bifurcated into the internal and the external carotid arteries.

\section{Discussion}

Transformations of the embryonic aortic arc system can cause the variations of the common carotid arteries. These transformations may affect the origins, the courses and the bifurcation levels of the vessels [2]. Absence of the common 

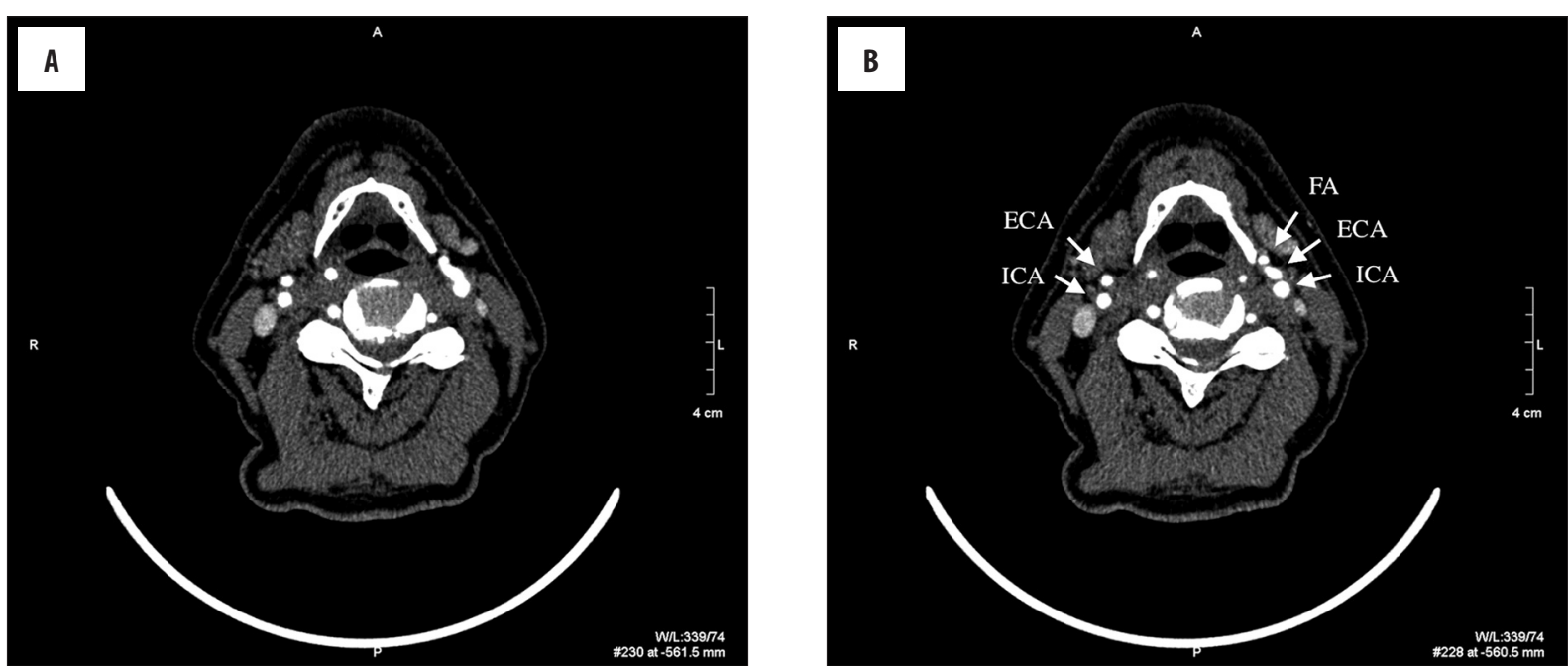

Figure 1. Axial images of the CT angiography (A, B) show trifurcation of the left common carotid artery.
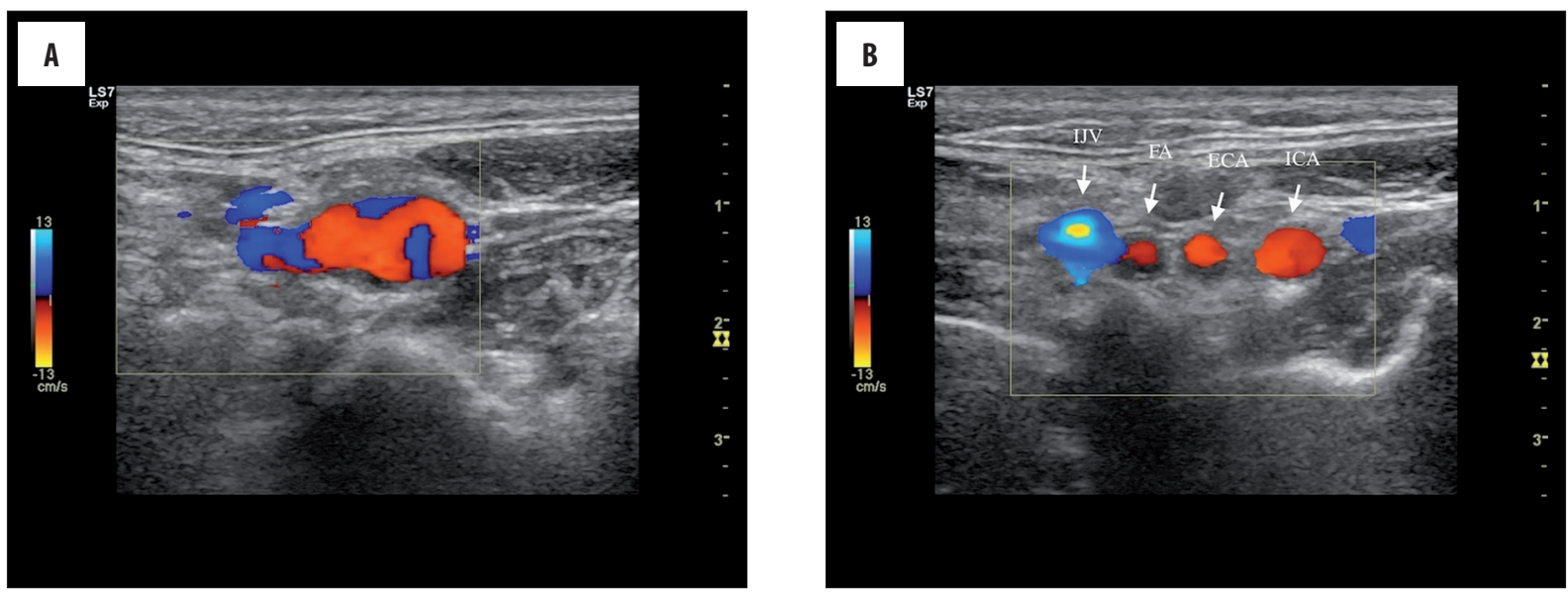

Figure 2. The sonographic images show trifurcation of the left common carotid artery at same level with axial CT images (A, B).

carotid artery, high or low bifurcation of the common carotid artery and the trifurcation of the common carotid artery can be seen as variations $[1,4]$. Normally the aortic arc divides into the brachiocephalic trunk, the left common carotid artery and the left subclavian artery. Then the brachiocephalic trunk terminates as the right common carotid artery and the right sublavian artery. The common carotid arteries course in the carotid sheath with the internal jugular vein and vagus nerve. At the both sides the common carotid arteries bifurcate into the internal and external carotid arteries at the level of fourth cervical vertebra. The external carotid artery supplies the arterial flow of the neck, face and base of skull. It gives six branches composed of the superior thyroid artery, ascending pharyngeal artery, lingual artery, facial artery, occipital artery and posterior auricular artery in the neck and terminates as the maxillary and superficial temporal arteries [1]. The internal carotid artery does not give any branches in the neck. It ascends and enters the carotid canal in the skull and gives the branches that supply the brain. In some cases, the common carotid artery gives rise to third artery, which can be a branch of the external carotid artery or the subclavian artery and forms the trifurcation of the common carotid artery [2]. In our case the left common carotid artery divided into the internal carotid, external carotid and facial arteries, and we demonstrated this variation via Doppler US examination and CT angiography.

During the surgical excision of carotid body tumors, which neuroendocrine tumors originating from the paraganglionic cells of the carotid bifurcation, preservation of the neuronal and vascular structures is of importance. Demir et al. reported patients with bilateral carotid body tumors whom developed vagal nerve palsy after unilateral surgical excision. They pointed that the major morbidity associated with surgery in these patients is related to postoperative cranial nerve dysfunction [5]. In this point of view, to avoid predictable surgical morbidity, the variations in carotid artery vessel anatomy also should be kept in mind.

The variations not only in the carotid artery system, but also in many vessels in the human body should be remembered. In a study by Sharma et al., congenital incomplete fusion of superior mesenteric artery (SMA) mimicking dissection was presented. In their case, while the initial CT revealed an abnormal appearance of the SMA as dissection, further investigation of the abdominal aortal and visceral arteries showed a congenital anatomical variant. They concluded that understanding the embryological origin of the abdominal vasculature combining with radiological signs 


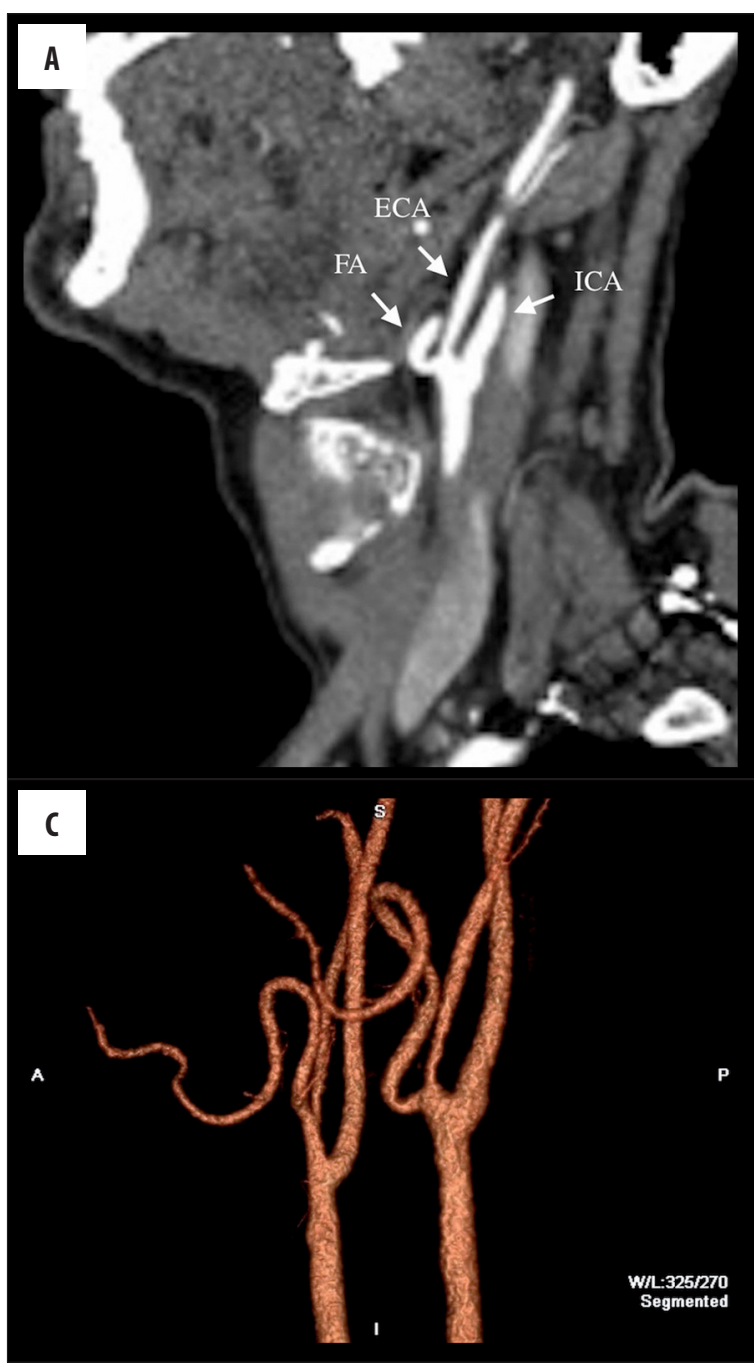

Figure 3. Sagittal reformat (A), MIP image (B), and 3-D reformat image $(\mathbf{C})$ of the $(\mathrm{T}$ angiography show the facial artery, the external carotid artery and the internal carotid artery arising from the common carotid artery.

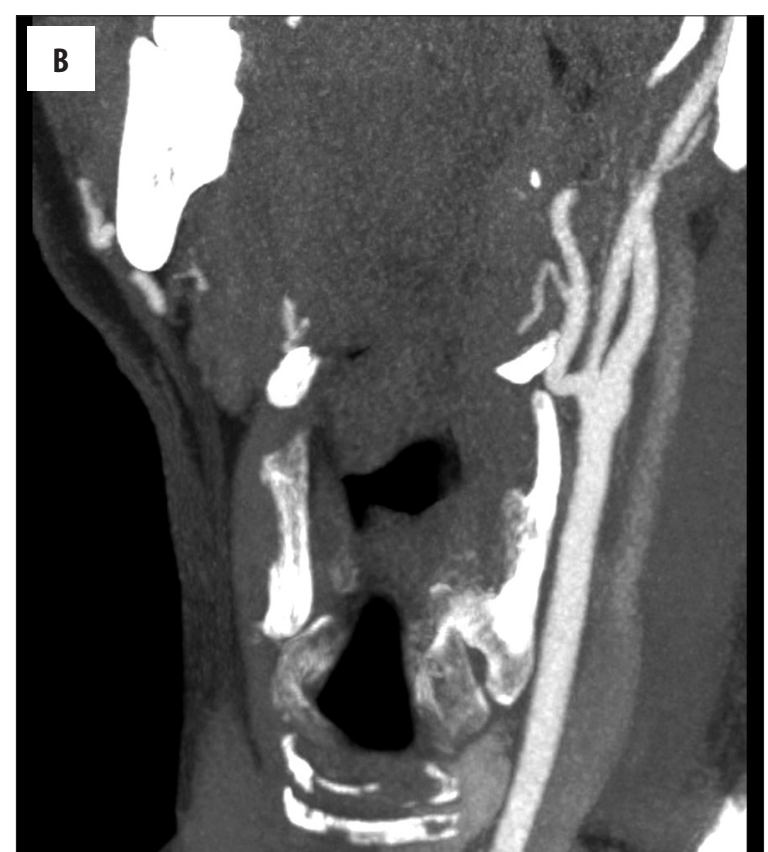

was important to distinguish variants from pathological findings [6]. As concomitant disorders or congenital anatomical variations may increase the risks of the surgery performed, the patient should be informed about the interventional carotid artery procedures (e.g. stenting, endarterectomy) and potential complications [7].

\section{Conclusions}

Because of the importance of the places where they supply blood, the variations of the carotid arteries should be known to avoid and reduce the complications during the invasive procedures.

\section{References:}

1. Chitra R: Trifurcation of the right common carotid artery. Indian $\mathrm{J}$ Plast Surg, 2008; 41: 85-88

2. Gürbüz J, Çavdar S, Özdoğmuş Ö: Trifurcation of the left common carotid artery: A case report. Clin Anat, 2001; 14: 58-61

3. Lucev N, Bobinac D, Maric I, Drescik I: Variations of the great arteries in the carotid triangle. Otolaryngol Head Neck Surg, 2000; 122: 590-91

4. Cakirer S, Karaarslan E, Kayabali M, Rozanes I: Separate origins of the left internal and external carotid arteries from the aortic arch: MR angiographic findings. Am J Neuroradiol, 2002; 23: 1600-2
5. Demir T, Uyar I, Demir HB et al: Five-year follow-up of a patient with bilateral carotid body tumors after unilateral surgical resection. Am J Case Rep, 2014; 15: 426-30

6. Sharma VK, H'ng MW: Congenital incomplete fusion of superior mesenteric artery mimicking dissection. Am J Case Rep, 2015; 16 : $41-44$

7. Stanišić MG, Rzepa T: Reasons underlying the consent to endovascular treatment, displayed by patients diagnosed with asymptomatic internal carotid artery stenosis. Med Sci Monit, 2014 20: 1503-9 